\title{
Study of environmental biodegradation of LDPE films in soil using optical and scanning electron microscopy
}

\begin{abstract}
An outdoor soil burial test was carried out to evaluate the degradation of commercially available LDPE carrier bags in natural soil for up to 2 years. Biodegradability of low density polyethylene films in soil was monitored using both optical and scanning electron microscopy (SEM). After 7-9 months of soil exposure, microbial colonization was evident on the film surface. Exposed LDPE samples exhibit progressive changes towards degradation after 17-22 months. SEM images reveal signs of degradation such as exfoliation and formation of cracks on film leading to disintegration. The possible degradation mode and consequences on the use and disposal of LDPE films is discussed.
\end{abstract}

Keyword: Biodegradation, LDPE, Optical microscopy, SEM, Soil burial 